\title{
Characterization and in vitro evaluation of a vaginal gel containing Lactobacillus crispatus for the prevention of gonorrhea
}

\author{
K.C. N'Guessan Gnaman ${ }^{\mathrm{a}, \mathrm{b}}$, S. Bouttier ${ }^{\mathrm{c}, 1}$, A. Yeo ${ }^{\mathrm{d}, 1}$, A.A.S. Aka Any-Grah ${ }^{\mathrm{b}, 1}$, S. Geiger ${ }^{\mathrm{a}, 1}$, \\ N. Huang ${ }^{\mathrm{a}}$, V. Nicolas ${ }^{\mathrm{e}}$, S. Villebrun ${ }^{\mathrm{a}}$, H. Faye-Kette ${ }^{\mathrm{d}}$, G. Ponchel ${ }^{\mathrm{a}}$, A.A. Koffi ${ }^{\mathrm{b}, 2}$, F. Agnely $^{\mathrm{a}, 2, *}$ \\ ${ }^{a}$ Université Paris-Saclay, CNRS, Institut Galien Paris-Saclay, 92290 Châtenay-Malabry, France \\ ${ }^{\mathrm{b}}$ Laboratoire de Pharmacie Galénique, Cosmétologie et Législation Pharmaceutique, UFR des Sciences Pharmaceutiques et Biologiques d'Abidjan, Université Félix \\ Houphouet-Boigny, 01 BP V 34 Abidjan 01, Côte d'Ivoire \\ ${ }^{\mathrm{c}}$ INRAE, AgroParisTech, MIcalis Institute, Équipe Bactéries pathogènes et santé, Université Paris-Saclay, 92290 Châtenay-Malabry, France \\ ${ }^{\mathrm{d}}$ Institut Pasteur, Departement de microbiologie, Unité des agents du tractus génital, Abidjan, Côte d'Ivoire \\ ${ }^{\mathrm{e}}$ Université Paris-Saclay, SFR-UMS-IPSIT, Plateforme d'imagerie cellulaire MIPSIT, 92290 Châtenay-Malabry, France
}

\section{A R T I C L E I N F O}

\section{Keywords:}

Confocal laser scanning microscopy

Gel

Gonorrhea prevention

Lactobacillus

Probiotics

Rheology

Vaginal administration

\begin{abstract}
A B S T R A C T
The increasing resistance of Neisseria gonorrhoeae to any current antibiotic treatment and the difficulties associated with the use of prevention means such as condom urge the need for alternative methods to prevent this sexually transmitted infection. In this work, a prevention strategy based on the use of a vaginal gel containing Lactobacilli was assessed in vitro. A Lactobacillus crispatus strain (ATCC 33197) was selected based on the published data on its ability to inhibit Neisseria gonorrhoeae. Its probiotic properties were first characterized. Then, a thermo-sensitive hydrogel containing $21.5 \%$ of poloxamer $407,1 \%$ of sodium alginate and $9 \log _{10} \mathrm{CFU}$ of Lactobacillus crispatus per gel sample $(5 \mathrm{~g})$ was developed. The gelation temperature and the rheological characteristics of this formulation appeared suitable for a vaginal administration. Lactobacillus crispatus was viable in the gel for six months although a large amount of the bacteria was not culturable. The ability of Lactobacillus crispatus to inhibit Neisseria gonorrhoeae was still observed with the gel. Such system, thus, appeared promising for the prevention of gonorrhea.
\end{abstract}

\section{Introduction}

Gonorrhea is a sexually transmitted infection that is caused by Neisseria gonorrhoeae. With 78 million new cases every year (WHO, 2016), it has become a major public health issue for many reasons. Indeed, this infection remains more often asymptomatic in women, which favors its spreading. It can induce severe damages such as infertility both in men and women, and blindness in newborns (Grodstein et al., 1993; Laga et al., 1986). It also increases the risk of contracting human immunodeficiency virus HIV (Cohen, 2004; Jarvis and Chang, 2012). Resistance to several antibiotics even to the last intention treatments is currently emerging (Lefebvre et al., 2018). Some untreatable strains that are insensitive to any known antimicrobial treatment are now circulating. Meanwhile, neither vaccines nor new drugs are emerging on the market. Condom which is the sole effective prevention mean is unfortunately either not well or insufficiently used. Thus, gonorrhea has become difficult to treat or to prevent. The main target of gonorrhea, as all sexually transmitted infections, is women because they are more vulnerable. A strategy giving women the control of their own protection could enable to achieve better results in the field of prevention (Stein, 1990). Thus, recent studies are devoted to woman-controlled means intended for a vaginal administration (Minnis and Padian, 2005; Traore et al., 2018).

Promising approaches based on probiotics have been developed for the prevention of women urogenital tract infections (Bruce and Reid, 1988; Stapleton et al., 2011). Probiotics are defined as "live

\footnotetext{
Abbreviations: A, sodium alginate; ATCC, american type culture collection; BHIA, brain heart infusion agar; CDC, Center for disease control; CFU, colony forming

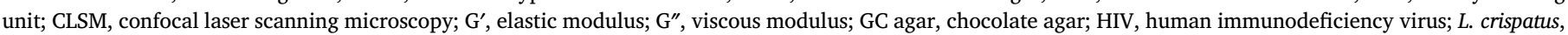

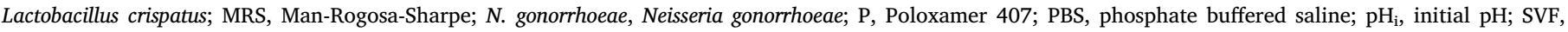
simulated vaginal fluid; $\mathrm{T}_{\text {gel }}$, gelation temperature; VNC, viable but nonculturable

* Corresponding author.

E-mail address: florence.agnely@universite-paris-saclay.fr (F. Agnely).

${ }^{1}$ These authors have contributed equally.

2 These authors have contributed equally.
} 
microorganisms that, when administered in adequate amounts, confer a health benefit on the host" (Hill et al., 2014). The most important probiotic genus studied for urogenital infections is Lactobacillus. The healthy vaginal flora is typically dominated by Lactobacillus $(L$.$) species,$ such as L. iners, L. crispatus, L. jensenii and L. gasseri (Pavlova et al., 2002; Ravel et al., 2011), but $L$. crispatus seems to largely prevail over the other Lactobacillus strains (Antonio et al., 1999; Vasquez et al., 2002). Moreover, L. crispatus has been reported to promote the stability of the normal vaginal flora (Verstraelen et al., 2009). Vaginal colonization with a human $L$. crispatus strain had been successful (Antonio and Hillier, 2003). A L. crispatus strain was also used successfully as a vaginal suppository for healthy women with a history of recurrent urinary tract infections (Czaja et al., 2007; Stapleton et al., 2011) and for the treatment of bacterial vaginoses (Hemmerling et al., 2010). Moreover, it was shown that L. crispatus inhibited the infectivity of N. gonorrhoeae in vitro (Amant et al., 2002) and ex vivo (Breshears et al., 2015). Its use for a vaginal administration could be a valuable strategy for vaginal gonorrhea prevention. Therefore, $L$. crispatus was selected in the present study. As the health promoting effects of probiotics are usually strain specific, we report, in this work, on the ability of a reference vaginal $L$. crispatus strain ATCC 33197 to be used as a probiotic for the prevention of gonorrhea.

The vaginal route has been selected instead of the oral route to obtain a maximal local effect while avoiding interferences with the gastrointestinal tract. Different strains of $L$. crispatus are already administered as capsules, vaginal suppositories or tablets (Nader-Macias and Juarez Tomas, 2015). However, these conventional dosage forms are associated to an inhomogeneous repartition in the vagina (Palmeira-de-Oliveira et al., 2015). In this study, we focused on the design of a gel form intended for the administration of this $L$. crispatus strain. Hydrogels are already used and well accepted for vaginal administration. They can easily spread over the vaginal mucosa, they are easy to be prepared and have a low cost of production (Cook and Brown, 2018, Pilcher, 2004). Besides, they can be self-administered without partner knowledge. Several studies have been focused on the formulation of viscoelastic and temperature-sensitive hydrogels based on poloxamer 407, with remanence capacity at the vaginal mucosa and sufficient resistance to dilution in presence of vaginal fluids (Aka-AnyGrah et al., 2010; Huang et al., 2016; Liu et al., 2009). In aqueous solution, this synthetic poly(ethylene oxide)-poly(propylene oxide)poly(ethylene oxide) triblock copolymer forms micelles above a critical micelle concentration or temperature. If the polymer concentration is high enough and above a critical temperature called gelation temperature $\left(\mathrm{T}_{\text {gel }}\right)$, these micelles organize themselves into a cubic liquid crystalline structure forming a gel (Dumortier et al., 2006). Poloxamer 407 can be mixed with mucoadhesive polymers such as cellulosic derivatives, polyacrylates or polysaccharides to reinforce remanence at the mucosal surface (Caramella et al., 2015, Rençber et al., 2017 Giuliano et al., 2018, Vigani et al., 2019). A clinical study conducted on 104 women investigated the vaginal administration of thermosentitive gels (polaxamer $407,17 \% \mathrm{w} / \mathrm{w}$, or poloxamer $407,15 \% \mathrm{w} / \mathrm{w}$, combined with hydroxyethyl cellose, $1 \% \mathrm{w} / \mathrm{w}$ ) containing sildenafil citrate (Soliman et al, 2017). Neither gel leakage nor side effects were reported. Among mucoadhesive polymers, sodium alginate is particularly attractive. Indeed, it is a natural polymer derived from brown algae that is commonly and widely used for drug delivery, cell encapsulation and immobilization (including Lactobacillus), wound dressing and enzymes immobilization (Chaluvadi et al. 2012, Lee and Mooney, 2012; Lin et al., 2004; Szekalska et al., 2016; Tønnesen and Karlsen, 2002). Poloxamer 407 and alginate are compatible (Grassi et al., 2006). Their mixture can be liquid at low or room temperature to allow easy administration and spreading on the vaginal surface, and then can undergo gelation at body temperature (Lin et al., 2004). Moreover, good tolerance and biocompatibility are expected. Indeed, a recent study showed that sodium alginate in oil-poloxamer organogels intended for vaginal administration of voriconazole were non-cytotoxic towards
HeLa and Vero cell lines (Querobino et al. 2019). Huang et al. (2016) also reported an absence of cytotoxicity in mouse fibroblast (L929) cell line of thermosensitive composite hydrogels based on poloxamer 407 and alginate used in transarterial embolization therapy. To the best of our knowledge, there is only one published study reporting the use of a gel for the administration of Lactobacilli into the vagina (Vigani et al., 2019). These authors developed two mixtures based on poloxamer 407 and methylcellulose loaded with Lactobacillus gasseri for preventing candidosis recurrences and they studied the viability of the probiotic over $24 \mathrm{~h}$.

In the present study, the mixture of poloxamer 407 and sodium alginate was assessed as a matrix for L. crispatus in order to obtain a temperature-sensitive vaginal gel with adequate mechanical properties and capable to inhibit $N$. gonorrhoeae. The probiotic properties of the selected $L$. crispatus strain were first assessed. In a second step, the influence of $L$. crispatus on the thermogelling and viscoelastic properties of the mixture of poloxamer 407 and alginate was investigated by rheology at $25{ }^{\circ} \mathrm{C}$, i.e. in administration conditions, and at $37{ }^{\circ} \mathrm{C}$ after dilution by simulated vaginal fluid (SVF) to mimic in vivo conditions after administration. The efficacy of the gel on $N$. gonorrhoeae was evaluated in vitro as well as the viability of the L. crispatus in the gel over a six-month period.

\section{Materials and methods}

\subsection{Materials}

L. crispatus, a reference strain, isolated from human vagina with collection numbers CIP 103603 (ATCC 33197; NCTC 4505; NCIMB 4505), and a reference strain $N$. gonorrhoeae with collection number CIP 107031 (ATCC 49226; CCM 4500; CCUG 26213; CDC 10.001; CNCTC 5194; DSM 9189) were purchased from Institut Pasteur (Paris). The strains were stored at $-80{ }^{\circ} \mathrm{C}$ in cryovials with the appropriate broth supplemented with $20 \%$ (v/v) glycerol. Streptococcus pneumoniae ATCC 49619 and Streptococcus pyogenes ATCC 12344 were obtained from Institut Pasteur (Abidjan). BD-Difco ${ }^{\mathrm{TM}}$ Lactobacilli of Man-Rogosa-Sharpe (MRS) broth or MRS agar, brain heart infusion agar and Columbia agar base were obtained from Fisher Scientific (France). Poloxamer 407 (P) (Lutrol ${ }^{\circledR}$ F127) of pharmaceutical grade was purchased from BASF (Germany). According to the supplier, the average molecular weight was $11774 \mathrm{~g} \cdot \mathrm{mol}^{-1}\left(9840-14600 \mathrm{~g} \cdot \mathrm{mol}^{-1}\right)$. Sodium alginate (A) was purchased from Roth (France), batch $\mathrm{N}^{\circ} 351172083$. Its weight-average molecular weight determined by size exclusion chromatography coupled to multi-angle light scattering was $300000 \mathrm{~g} \cdot \mathrm{mol}^{-1}$. The M:G ( $\beta$ D-mannuronic acid: $\alpha$-L-guluronic acid) ratio determined by ${ }^{1} \mathrm{H}$ NMR was $1.24 \pm 0.04$. Bovine serum albumin and all other reagents were supplied by Sigma-Aldrich (France) and were of analytical grade. Live/ dead $^{\circledR}$ bacLight $^{\mathrm{TM}}$ bacterial viability kit was purchased from Fisher Scientific (France). Sterile ultrapure water (resistivity 18.2 M 2 , MilliQ, Millipore, France) was used. All the antibiotic discs were from BIO-RAD (France) and the E-test from Biomérieux (France).

\subsection{Study of Lactobacillus crispatus}

\subsubsection{Growth kinetic}

L. crispatus $(1 / 1000 \mathrm{v} / \mathrm{v})$ in MRS broth was incubated at $37{ }^{\circ} \mathrm{C}, 5 \%$ $\mathrm{CO}_{2}$ to determine its stationary growth phase. Then, the number of colony-forming units was determined at the beginning $\left(\mathrm{t}_{0}\right)$ and every three hours for $24 \mathrm{~h}$, using a MRS agar plate. This test was performed in triplicate in one experiment.

\subsubsection{Acid production and growth at different $p H$}

The evaluation of the acidification ability of $L$. crispatus and of the effects of low $\mathrm{pH}$ on its growth was achieved according to Boskey et al. (1999), with slight modifications. MRS broth was prepared and adjusted to specific initial $\mathrm{pH}\left(\mathrm{pH}_{\mathrm{i}}\right)$ ranging from 4 to 7 with either $\mathrm{HCl}$ 
or $\mathrm{NaOH}$ before autoclaving. $L$. crispatus was inoculated $(1 \% \mathrm{v} / \mathrm{v})$ into $10 \mathrm{~mL}$ of each MRS broth giving a starting concentration of about $10^{7}$ cells $/ \mathrm{mL}$. These suspensions were incubated for $24 \mathrm{~h}$ at $37^{\circ} \mathrm{C}$ under $5 \% \mathrm{CO}_{2}$. The amount of acid produced by $L$. crispatus was indirectly determined by measuring the $\mathrm{pH}$ immediately after preparation $\left(\mathrm{t}_{0}\right)$ and after a $24 \mathrm{~h}$ incubation $\left(\mathrm{t}_{24 \mathrm{~h}}\right)$ with a $\mathrm{pH}$ meter (Metter Toledo equipped with an electrode Seven2GO, France). To assess the sensitivity of L. crispatus to the $\mathrm{pH}$, the numbers of $\mathrm{CFU} / \mathrm{mL}$ of each MRS broth were quantified at the beginning $\left(\mathrm{t}_{0}\right)$ and after $24 \mathrm{~h}\left(\mathrm{t}_{24 \mathrm{~h}}\right)$. This assay was performed in two separate experiments constituted of three replicates each.

\subsubsection{Auto-aggregation and co-aggregation assays}

This test was conducted to appreciate the ability of $L$. crispatus to auto-aggregate and co-aggregate $N$. gonorrhoeae. It was performed according to Verdenelli et al. (2014) with slight modifications. The cultures were harvested by centrifugation $\left(2800 \mathrm{~g}, 10 \mathrm{~min}, 4^{\circ} \mathrm{C}\right)$, and washed twice with sterile water (pH7). Bacterial suspensions in water of $L$. crispatus and $N$. gonorrhoeae were adjusted to $0.5 \mathrm{McFarland}$. Equal volumes $(1 \mathrm{~mL})$ of each suspension were mixed for $20 \mathrm{~s}$ using a vortex and left for $4 \mathrm{~h}$ at $37{ }^{\circ} \mathrm{C}$. A droplet of the single suspensions and the mixture was then put on a glass slide immediately and after four hours. After Gram-staining, aggregates, defined as visible clumps of bacteria, were observed using a B-290 TB Optika microscope (Italy). This experiment was performed in triplicate.

\subsubsection{Hemolytic activity}

To evaluate the ability of $L$. crispatus to induce hemolysis, the hemolytic activity of L. crispatus was determined according to Maragkoudakis et al. (2009) with slight modifications. In short, the subculture of L. crispatus of $16 \mathrm{~h}$ in MRS broth was streaked onto Columbia agar base plates supplemented with $5 \%(\mathrm{v} / \mathrm{v})$ whole sheep blood. The plates were incubated at $37{ }^{\circ} \mathrm{C}$ under $5 \% \mathrm{CO}_{2}$ for $24 \mathrm{~h}$ and $48 \mathrm{~h}$ in anaerobic conditions. Then, the clear zones and the color of hemolysis around the growth colonies were observed. Streptococcus pneumoniae ATCC 49619 and Streptococcus pyogenes ATCC 12344 were used as controls for respectively $\alpha$ - and $\beta$-hemolysis. This experiment was performed thrice.

\subsubsection{Antibiotic susceptibility profile}

L. crispatus susceptibility to different classes of antibiotics was evaluated by disc ( $6 \mathrm{~mm}$ diameter) method. At least two independent sets of experiments were performed. As a control to the disc method, susceptibility to azithromycin, cefixime, ceftriaxone, ciprofloxacin, gentamicin, penicillin $\mathrm{G}$ and tetracycline was also evaluated by the Etest method according to the manufacturer's instructions. This method provides a convenient and quantitative test of antibiotic resistance. All the plates were incubated for 24 and $48 \mathrm{~h}$. MRS agar was used in order to favor the growth of L. crispatus.

\subsection{Purity control and counting of L. crispatus}

Purity was assessed by macroscopic observation of colonies and microscopic observation after Gram staining. The serial decimal dilution method, by plating onto MRS agar medium, was used for counting total Lactobacilli in every suspensions and gels. Dilutions were made in PBS and after vortexing each dilution for $5 \mathrm{~s}, 100 \mu \mathrm{L}$ of the previous dilution were inoculated in $900 \mu \mathrm{L}$ of the next dilution. Then, $100 \mu \mathrm{L}$ of the chosen dilutions were poured on the MRS agar plates.

\subsection{Hydrogel preparation}

A concentrated mixture of polymers was prepared. Polymer solubilization was performed by using a mixer equipped with a turbine adapted to the mixing of viscous preparations (Rayneri-turbotest, Rayneri, France) under an agitation of $1000 \mathrm{rpm}$ as
Table 1

Hydrogels containing alginate (A), poloxamer (P) with or without L. crispatus (L).

\begin{tabular}{llll} 
Acronyms & Poloxamer $(\% \mathrm{w} / \mathrm{w})$ & Alginate $(\% \mathrm{w} / \mathrm{w})$ & L. crispatus $\left(\log _{10} \mathrm{CFU} / 5 \mathrm{~g}\right.$ of gel) \\
\hline P21.5A1 & 21.5 & 1 & - \\
P21.5A1L & 21.5 & 1 & 9 \\
\hline
\end{tabular}

described by Aka-Any-Grah et al. (2010). The alginate powder (1.250 g) was gradually added under agitation at room temperature to ultrapure water (71.875 g). After complete dissolution of alginate, the poloxamer powder $\left(26.875 \mathrm{~g}\right.$ ) was gradually added at $4{ }^{\circ} \mathrm{C}$ under the same agitation. After complete dissolution, this mixture was equilibrated $48 \mathrm{~h}$ at $4{ }^{\circ} \mathrm{C}$ to eliminate air bubbles and was autoclaved at $121{ }^{\circ} \mathrm{C}$, 15 min before its mixing with the Lactobacilli suspension.

Meanwhile, frozen L. crispatus $\left(-80^{\circ} \mathrm{C}\right)$ was isolated on MRS agar for 48-72 h. Then, it was subcultured twice, first for $24 \mathrm{~h}$ and second for $16 \mathrm{~h}\left(37{ }^{\circ} \mathrm{C}, 5 \% \mathrm{CO}_{2}\right)$. This second subculture was centrifuged ( $2800 \mathrm{~g}, 10 \mathrm{~min}, 4^{\circ} \mathrm{C}$ ) and washed twice with sterile water before being suspended in sterile water. L. crispatus homogenization was achieved by vortexing. L. crispatus suspension was enumerated by plating serial dilutions onto MRS agar.

Final gels with or without $L$. crispatus were obtained by mixing respectively $1 \mathrm{~mL}$ of sterile water or $1 \mathrm{~mL}$ of the $L$. crispatus suspension to $4 \mathrm{~g}$ of the concentrated polymer mixture. This allowed obtaining two types of gels (P21.5A1 and P21.5A1L) whose final compositions are presented in Table 1. P21.5A1L contained $9 \log _{10} \mathrm{CFU}$ of $L$. crispatus per gel sample (5 g).

\subsection{Preparation of hydrogels diluted by simulated vaginal fluid}

Previous studies showed that $5 \mathrm{~mL}$ of gels were able to properly cover the human vaginal epithelium, immediately after insertion, within the first $30 \mathrm{~min}$ and without sexual intercourse (Barnhart et al., 2004). The volume of ambient fluid present in the vagina at any time is approximately 0.5-0.75 mL (Owen and Katz, 1999). Therefore, to mimic the maximum dilution likely to occur in the vagina after the gel administration, $5 \mathrm{~mL}$ of each formulation were diluted with $0.75 \mathrm{~mL}$ of simulated vaginal fluid (SVF). SVF containing sodium chloride, potassium hydroxide, calcium hydroxide, bovine serum albumin, lactic acid, acetic acid, glycerol, urea and glucose was prepared according to Owen and Katz (1999). It simulates the vaginal fluid from healthy, nonpregnant, premenopausal women and can be used to study vaginal formulations in vitro. P21.5A1 + SVF and P21.5A1L + SVF denoted respectively $\mathrm{P} 21.5 \mathrm{~A} 1$ and $\mathrm{P} 21.5 \mathrm{~A} 1 \mathrm{~L}$ samples diluted with SVF. The aim of this test was to assess the influence of the dilution by SVF on the thermogelling and the rheological properties of the formulations.

\subsection{Rheological characterization of the hydrogels}

The rheological properties of the gels were assessed at $25{ }^{\circ} \mathrm{C}$, at $37{ }^{\circ} \mathrm{C}$ and after dilution of the gels by SVF at $37{ }^{\circ} \mathrm{C}$. Experiments were conducted on a rotational rheometer ARG2 (TA instruments, New Castle, USA) equipped with an aluminum cone/plate geometry (diameter $40 \mathrm{~mm}$, angle $1^{\circ}$ and cone truncation $28 \mu \mathrm{m}$ ) and with a solvent trap to limit evaporation during measurement. Peltier diodes, placed in the lower plate, allowed controlling the temperature. The samples were equilibrated $3 \mathrm{~min}$ before starting the experiments. In flow measurements, the viscosity $(\eta)$ was recorded as a function of the shear rate. The shear rate was increased gradually from $0.01 \mathrm{~s}^{-1}$ to $1000 \mathrm{~s}^{-1}$ over $3 \mathrm{~min}$, maintained at $1000 \mathrm{~s}^{-1}$ for $1 \mathrm{~min}$ and then decreased gradually from $1000 \mathrm{~s}^{-1}$ to $0.01 \mathrm{~s}^{-1}$ over $3 \mathrm{~min}$. In oscillatory measurements, linear viscoelastic regions, where the moduli remained constant as the shear stress increased, were determined. The gelation temperature $\left(\mathrm{T}_{\text {gel }}\right)$ and viscoelastic properties of the gels were 
assessed under a stress value belonging to their viscoelastic linear regime. A temperature sweep from 5 to $45^{\circ} \mathrm{C}$ at a rate of $1{ }^{\circ} \mathrm{C} / \mathrm{min}$ was performed at a frequency of $1 \mathrm{~Hz}$, and the elastic modulus $\left(\mathrm{G}^{\prime}\right)$ as well as the viscous modulus $\left(\mathrm{G}^{\prime \prime}\right)$ were recorded as a function of the temperature. The gelation temperature $\left(\mathrm{T}_{\text {gel }}\right)$ was determined as the temperature of the crossover of $\mathrm{G}^{\prime}$ and $\mathrm{G}^{\prime \prime}$. $\mathrm{G}^{\prime}$ and $\mathrm{G}^{\prime \prime}$ were also recorded during frequency sweeps from 0.01 to $50 \mathrm{~Hz}$. These experiments were performed in triplicate.

\section{7. $\mathrm{pH}$ measurement of the gels}

$\mathrm{pH}$ of the gels was measured at room temperature with a $\mathrm{pH}$-meter Mettler Toledo Seven2Go, suited for measurements in viscous aqueous media. This experiment was performed in triplicate.

\subsection{Assessment of the distribution of L. crispatus within the gel}

The distribution and viability of $L$. crispatus within the gels were assessed by using Syto9 that stained both living and dead bacteria in green and propidium iodide that stained only dead bacteria in red. When used simultaneously, Syto9 seemed to allow the observation of living bacteria in green, and propidium iodide the observation in red of the dead ones (Moore et al., 2015). These fluorescent stains were introduced at $1 / 1000(\mathrm{v} / \mathrm{w})$ in the gels. In short, they were introduced in the 1-mL L. crispatus suspension before mixing it with the gels in the liquid state. Then, they were immediately observed by microscope. This study was performed with an inverted confocal laser scanning microscope LSM 510-Meta (Carl Zeiss, Germany) using a Plan-Apochromat $63 \mathrm{X} / 1.4$ objective lens, equipped with an argon (488 nm excitation wavelength) and a helium neon laser (543 nm excitation wavelength). The green and the red fluorescence emissions were collected with a 505-550 nm band-pass and a $560 \mathrm{~nm}$ long pass emission filter respectively, under a sequential mode. The pinhole was set at 1.0 Airy unit. For each condition, single 2D images were collected as well as 3D images with an image every $0.9 \mu \mathrm{m}$ along the $\mathrm{z}$ axis to reach a thickness of $32 \mu \mathrm{m}$ taken arbitrarily. 12 bit numerical images were acquired with LSM 510 software version 3.2.

\subsection{Stability studies of the gel P21.5A1L}

P21.5A1L was stored over six months at 4 and $25{ }^{\circ} \mathrm{C}$, respectively below and above the gelation temperature, in order to assess its stability. The gel viscosity at a shear rate of $125 \mathrm{~s}^{-1}$ and $\mathrm{pH}$ were measured as previously described. Counting on MRS agar plate was performed to investigate the culturability of cells. In brief, after serial decimal dilutions, three dilutions were plated on MRS agar. Plates were incubated at $37^{\circ} \mathrm{C}$ for $48 \mathrm{~h}$ before counting the colony forming units. Otherwise, $L$. crispatus distribution and viability were evaluated by confocal laser scanning microscopy. Briefly, the fluorescent stains (Syto9 and propidium iodide) were introduced at $1 / 1000(\mathrm{v} / \mathrm{w})$ in the liquid gels containing $L$. crispatus, vortexed and immediately observed by confocal microscopy as previously described in Section 2.8.

\subsection{Inhibition of the growth of N. gonorrhoeae}

For the preparation of $N$. gonorrhoeae suspension, the frozen stock was isolated on Thayer-Martin medium plate and incubated for $18 \mathrm{~h}$ at $37{ }^{\circ} \mathrm{C}$ in a moist atmosphere enriched with $5 \% \mathrm{CO}_{2}$. These conditions were used because autolysis may occur during prolonged incubation, and it may be difficult to obtain a suspension from growth on agar plates. Then, colonies were suspended in $\mathrm{NaCl} 0.85 \%$ to a turbidity equivalent to $0.5 \mathrm{McF}$ arland standard. These suspensions contained $10^{6} \mathrm{~N}$. gonorrhoeae cells/mL. They were mixed thoroughly using a vortex mixer to break up clumps of growth.

The inhibition of $N$. gonorrhoeae was evaluated by using a modified version of the agar overlay technique of Amant et al. (2002). Fig. 1

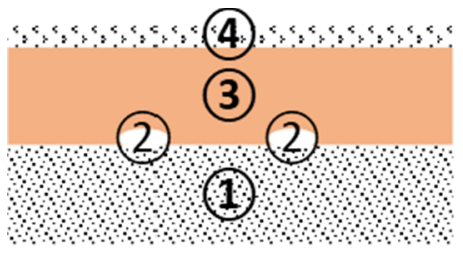

Incubation $24 \mathrm{~h}, 37^{\circ} \mathrm{C}$

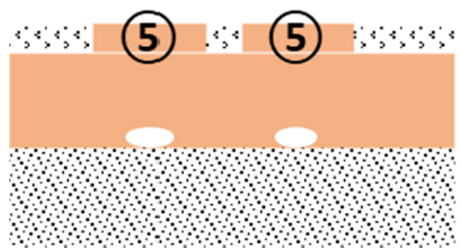

Fig. 1. Schematic depiction of the inhibition of $N$ gonorrhoeae test: (1) brain heart infusion agar at pH 5 or 6.5 , (2) spots of gels or suspensions containing L. crispatus after incubation $24 \mathrm{~h}, 37^{\circ} \mathrm{C}$, (3) chocolate agar medium, (4) spreading of $N$. gonorrhoeae, (5) inhibition zones.

presents the method schematically. Brain heart infusion agars (BHIA) with $\mathrm{pH}$ adjusted either to 5 or 6.5 were prepared before being autoclaved at $121{ }^{\circ} \mathrm{C}$ over $15 \mathrm{~min}$. Gels or suspensions containing L. crispatus were inoculated onto BHIA plates (60 mm diameter), using respectively a sterile handle or a micropipette to obtain spots of $0.5 \mathrm{~g}$ for the gels or of $50 \mu \mathrm{L}$ for the suspensions. After $24 \mathrm{~h}$ of incubation, $4 \mathrm{~mL}$ of chocolate agar (containing proteose peptone, dextrose, sodium chloride, disodium phosphate, agar, hemoglobin) were poured onto the BHIA plates and allowed to solidify. Then, $500 \mu \mathrm{L}$ of $N$. gonorrhoeae suspension were spread onto the agar overlay and incubated for $24 \mathrm{~h}$. The presence of a zone of growth inhibition was considered as positive for inhibition. Control experiments were performed by using the same protocol without the step of spot formation. Moreover, the effect of a gel without L. crispatus was also assessed. For all experiments, the number of L. crispatus in the suspensions or the gels was confirmed by standard serial dilution and culture. The thickness of each agar layer was about $2 \mathrm{~mm}$.

\section{Results and discussion}

In this study, a well identified and characterized reference strain that originated from a human vagina and that had already shown its efficacy on $N$. gonorrhoeae (Amant et al., 2002; Breshears et al., 2015) was selected. Its probiotic benefits such as its acidification ability and its capacity to auto-aggregate or co-aggregate $N$. gonorrhoeae were first confirmed before formulation development and in vitro efficacy studies.

\subsection{Characterization of L. crispatus}

Based on published data, L. crispatus suspensions containing $9 \log _{10} \mathrm{CFU} / \mathrm{mL}$ were selected. Amant et al. (2002) showed the effectiveness of different dilutions of $10^{8} \mathrm{~L}$. crispatus on $N$. gonorrhoeae and Reid et al. (2001) showed the effectiveness of over $10^{8}$ viable probiotic per day to restore and maintain a normal vaginal flora. An expert consensus document stated that the minimal number of viable cells should be $1 \times 10^{9} \mathrm{CFU}$ administered per day (Hill et al., 2014).

L. crispatus growth kinetic test allowed determining its stationary phase that began after $9 \mathrm{~h}$ of culture. Based on this result, for all the tests, cultures of $16 \mathrm{~h}$ of incubation were used to ensure reproducible physiological and metabolic states of the strain.

As regards L. crispatus acidification ability, this strain was grown in either standard growth medium $\left(\mathrm{pH}_{\mathrm{i}} 6.5\right)$ or in modified $\mathrm{pH}$ growth medium $(4,5,6,7)$. The use of these $\mathrm{pH}$ values was based on the fact 
Table 2

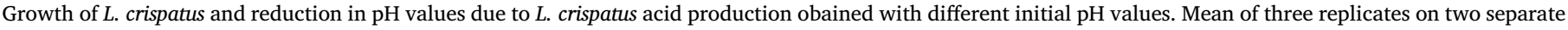
experiments.

Initial $\mathrm{pH}$

Increase in the number of $L$. crispatus at $24 \mathrm{~h}, 37^{\circ} \mathrm{C}, 5 \% \mathrm{CO}_{2}\left(\log _{10} \mathrm{CFU} / \mathrm{mL}\right)$

Reduction in $\mathrm{pH}$ due to $L$. crispatus at $24 \mathrm{~h}, 37^{\circ} \mathrm{C}, 5 \% \mathrm{CO}_{2}$

$\begin{array}{lll}4.00 \pm 0.01 & 5.00 \pm 0.01 & 6.00 \pm 0.01 \\ 1.19 \pm 0.23 & 1.57 \pm 0.41 & 1.85 \pm 0.46 \\ 0.74 \pm 0.17 & 1.49 \pm 0.13 & 1.96 \pm 0.02\end{array}$

$\begin{array}{lll}1.19 \pm 0.23 & 1.57 \pm 0.41 & 1.85 \pm 0.46 \\ 0.74 \pm 0.17 & 1.49 \pm 0.13 & 1.96 \pm 0.02\end{array}$

$6.50 \pm 0.01$

$1.88 \pm 0.11$

$2.24 \pm 0.08$ $\pm 0.01$

$2.00 \pm 0.16$

$2.39 \pm 0.04$ that vaginal $\mathrm{pH}$ varies between 3.5 and 5.5 and increases up to 7 in the presence of seminal fluid (O'Hanlon et al., 2013; Owen and Katz, 1999). Therefore, the growth and the acid production of $L$. crispatus were simultaneously evaluated in MRS at $\mathrm{pH}$ ranging from 4 to 7 to mimic this vaginal $\mathrm{pH}$. In MRS, the Lactobacilli had the nutrients they needed to grow and $\mathrm{pH}$ was the only modified parameter in order to assess its influence. The growth of $L$. crispatus was favored at $\mathrm{pH}$ ranging from 6 to 7 (Table 2). This $\mathrm{pH}$ range corresponded to the $\mathrm{pH}$ of the mixture of polymers, which should thus be favorable for $L$. crispatus addition. Otherwise, whatever the $\mathrm{pH}_{\mathrm{i}}$ of MRS, a decrease in $\mathrm{pH}$ values was observed after $24 \mathrm{~h}$ of incubation at $37{ }^{\circ} \mathrm{C}$ and $5 \% \mathrm{CO}_{2}$, mimicking the vaginal conditions. The variations of $\mathrm{pH}$ are given in Table 2 . They were also higher at $\mathrm{pH}$ varying between 6 and 7, suggesting a correlation between $L$. crispatus viability and acid production. A reduction of $\mathrm{pH}$ by L. crispatus indicated indirectly an acidification due to lactic acid production. Thus, this result suggested higher lactic acid production at $\mathrm{pH}$ higher than 6 . Both $\mathrm{pH}$ and lactic acid concentration of a media may inactivate pathogens individually or in combination. O'Hanlon et al. (2013) showed that as the alkaline $\mathrm{pH}$ of the vagina due to seminal fluid reduced the protective action of vaginal acidity against pathogens, the level of vaginal lactic acid was likely to reduce the transmission of vaginal acid-sensitive pathogens. As only protonated lactic acid might have microbicidal activity, it would be better to have a pH below the pKa of lactic acid (3.90) (Tachedjian et al., 2017). In this study, the quantity of protonated lactic acid was not measured, but as the final $\mathrm{pH}$ in each sample was between 3.1 and 3.9, it can be supposed that lactic acid was in its protonated state favorable for its antibacterial activity. The final $\mathrm{pH}$ in each sample was comparable to the $\mathrm{pH}$ of healthy vagina dominated by Lactobacilli as described by O'Hanlon et al. (2013).

Moreover, Graver and Wade (2011) showed the important role of acidification in the inhibition of $N$. gonorrhoeae. For Foschi et al. (2017), the presence of lactate seemed to be crucial for the anti-gonococcal activity, especially for $\mathrm{pH}$ values ranging from 4.4 to 5.3 , indicating that the presence of hydrogen ions was necessary but not sufficient to inhibit gonococci. In our study, results of the acid production and the growth of L. crispatus in the $\mathrm{pH}$ range of 4 to 7 predicted a favorable growth in the vagina containing natural nutrients and favorable conditions for the inhibition of $N$. gonorrhoeae (see Section 3.5). Amant et al. (2002) also showed the ability of this Lactobacillus strain to inhibit $N$. gonorrhoeae both at acid and neutral pH. Based on these studies, the higher reduction in $\mathrm{pH}$ related to higher lactic acid production was considered as more interesting.

The auto-aggregation and co-aggregation with $N$. gonorrhoeae properties of L. crispatus were investigated. Fig. 2 shows their organization in suspension after a $4 \mathrm{~h}$-incubation time in suspension. L. crispatus formed large and dense visible clumps (Fig. 2A) whereas N. gonorrhoeae formed a clump similar to that observed by Anderson et al. (2016) (Fig. 2C), with an aspect different than that of $L$. crispatus (Fig. 2A). Small and widely distributed aggregates were observed in the mixtures of $L$. crispatus and $N$. gonorrhoeae (Fig. 2B). They were arranged as microcolonies similar to the ones observed by Foschi et al. (2017). L c crispatus can change its organization in the presence of pathogens to co-aggregate with them. Reid et al. (1990) showed that when Lactobacilli formed co-aggregates and bound to pathogens, this resulted in returns to homeostasis, because co-aggregation created a hostile biochemical micro-environment around the pathogen and prevented it from continuation of growth and domination of the niche.

Regarding the security aspect associated with the use of this $L$. crispatus strain, two parameters were studied, its hemolytic activity and its antimicrobial resistance. Hemolytic activity test showed that the Columbia sheep blood agar aspect around the colonies was unchanged, meaning $L$. crispatus did not exhibit hemolysis. It is a non-hemolytic bacterium. This result was in accordance with many reports (Ambalam et al., 2012; Kassaa et al., 2014; Maragkoudakis et al., 2006). The antibiotic resistance is another key factor for probiotics as the level of susceptibility to the antimicrobial agents is species-dependent. Table 3 presents the results of the disc method. The E-test and the disc method gave similar results for the antibiotics studied by both methods. The resistance to gentamicin and ciprofloxacin was observed both by E-test and disc diffusion methods. Results showed that L. crispatus was resistant to an aminoglycoside (gentamicin), quinolones (ciprofloxacin, nalidixic acid, pefloxacin) and a lincosamide (clindamycin) (Table 3). Several studies have reported intrinsic resistance of Lactobacilli to quinolones, gentamicin, and other antibiotics (Danielsen and Wind, 2003;
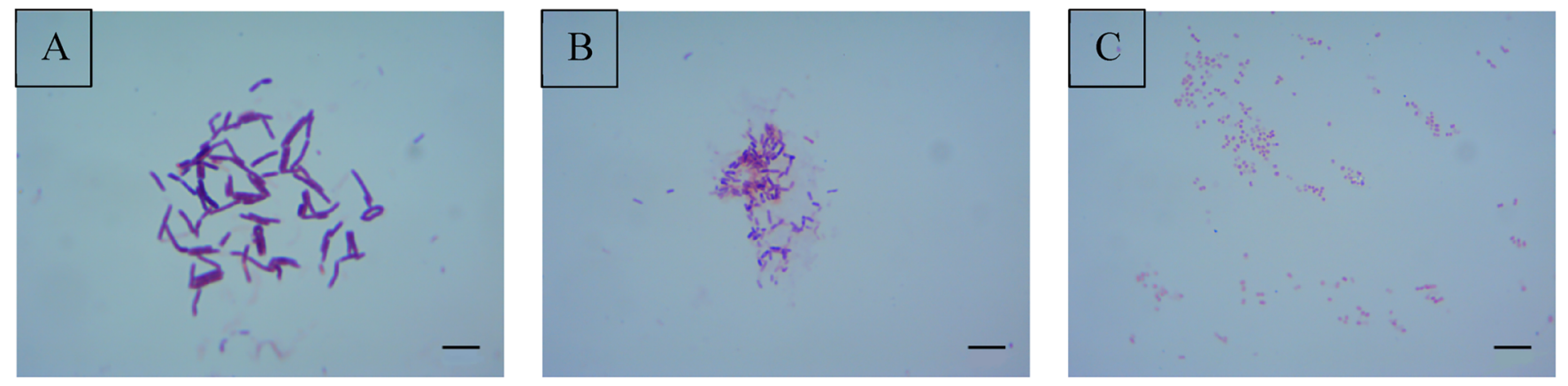

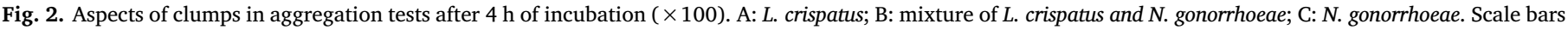
correspond to $10 \mu \mathrm{m}$. 
Štšepetova et al., 2017; Temmerman et al., 2003). According to the European Food Safety Authority (EFSA) guidance on the assessment of bacterial susceptibility to antimicrobials of human and veterinary importance, bacterial strains carrying intrinsic resistance present a minimal risk for horizontal spread and thus, may be used as a food additive. Based on these in vitro tests, L. crispatus is an interesting probiotic candidate. However, further studies should be performed to search for pathogenicity genes, as well as in vivo studies to confirm that this $L$. crispatus strain would not induce any pathogenicity and could be regarded as a safe microorganism for a vaginal administration.

\subsection{Preparation and rheological characterization of gels}

In a second step of our study, L. crispatus was incorporated in an aqueous mixture of poloxamer 407 and sodium alginate in order to develop a formulation with characteristics that were suitable for a vaginal administration. In a preliminary optimization step, the concentration of poloxamer $407(21.5 \%)$ and sodium alginate $(1 \%)$ were selected in order to obtain a formulation that remained in gel state at $37{ }^{\circ} \mathrm{C}$, even after dilution with SVF, with minimal polymer concentrations. Thereafter, the study focused on this gel denoted P21.5A1 and on its corresponding gel (P21.5A1L) containing L. crispatus at $9 \log _{10} \mathrm{CFU}$ per gel sample $(5 \mathrm{~g})$. The counting of Lactobacilli in both suspensions and gels after their preparation showed that, in the gel, it represented $1 / 5$ of the number in the suspension. That corresponded to the dilution ratio of $L$. crispatus suspension $(1 \mathrm{~mL})$ with the gel $(4 \mathrm{~g})$. This result suggested that the fabrication process was not deleterious for the Lactobacilli.

Both gels were characterized by rheology (Fig. 3, Table 4). The gel without L. crispatus, P21.5A1, showed a shear-thinning behavior (Fig. 3A, B). This behavior should allow an easy administration. This formulation was also non-thixotropic even after dilution by the simulated vaginal fluid (Fig. 3A, B). Therefore, as soon as the shear was removed, the viscosity returned to its initial high value, which should be favorable to maintain the gel at the administration site and to increase the residence time of the formulation in the vagina. P21.5A1 also had a thermogelling behavior (Fig. 3C). Below $\mathrm{T}_{\text {gel }}$, the elastic modulus $\left(G^{\prime}\right)$ was lower than the viscous modulus $\left(G^{\prime \prime}\right)$ revealing a dominant viscous behavior. Above $T_{\text {gel }}, G^{\prime}$ was higher than $G^{\prime \prime}$ showing a dominant elastic behavior. P21.5A1 gelation temperature was $19 \pm 1{ }^{\circ} \mathrm{C}$. After dilution with SVF, $\mathrm{T}_{\text {gel }}$ was increased (Fig. 3C, D, Table 4) whereas the viscoelastic moduli values decreased due to the diminution of the concentration of poloxamer (Fig. $3 \mathrm{C}$ versus $\mathrm{D}$ and $\mathrm{E}$ versus F, Table 4). Aka-Any-Grah et al. (2010) also observed this phenomenon. Interestingly, when $L$. crispatus was introduced into the gel $(\mathrm{P} 21.5 \mathrm{~A} 1 \mathrm{~L})$, the rheological properties were similar to those of P21.5A1 (Fig. 3, Table 4). Thus, $L$. crispatus did not significantly affect the rheological properties of this mixture of poloxamer 407 and sodium alginate, even after dilution with SVF.

Table 3

Antibiotics susceptibility of L. crispatus by disc diffusion method as a mean of at least two repetitions, showing highlighted in gray the antibiotics to which L. crispatus was resistant.

\begin{tabular}{|c|c|c|c|}
\hline Antibiotic discs (dose/disc) & $\begin{array}{l}\text { Inhibition zone } \\
\text { diameter }(\mathrm{mm})\end{array}$ & Antibiotic discs (dose/disc) & $\begin{array}{l}\text { Inhibition zone } \\
\text { diameter (mm) }\end{array}$ \\
\hline PENICILLIN CLASS & & TETRACYCLINES CLASS & \\
\hline Penicillin G $(10 \mu \mathrm{g})$ & 26 & Doxycycline (DOX, $30 \mu \mathrm{g})$ & 26 \\
\hline Ampicillin (AMP, $10 \mu \mathrm{g})$ & 25 & Minocycline (MN, $30 \mu \mathrm{g})$ & 29 \\
\hline Amoxicillin (AMX, $25 \mu \mathrm{g}$ ) & 24 & Tetracycline (TET, $30 \mu \mathrm{g}$ ) & 28 \\
\hline Oxacillin (OXA, $1 \mu \mathrm{g})$ & 27 & MACROLIDES CLASS & \\
\hline Piperacillin (PIP, $75 \mu \mathrm{g}$ ) & 34 & Azithromycin (AZM, $15 \mu \mathrm{g})$ & 20 \\
\hline Ticarcillin (TIC, $75 \mu \mathrm{g}$ ) & 37 & Spiramycin (SPN, $100 \mu \mathrm{g})$ & 18 \\
\hline Amoxicillin-clavulanic acid (AMC, $20 / 10 \mu \mathrm{g})$ & 27 & Erythromycin (ERY, $15 \mu \mathrm{g})$ & 30 \\
\hline Piperacillin-tazobactam (PPT, 75/10 $\mu \mathrm{g})$ & 24 & CHLORAMPHENICOL CLASS & \\
\hline Ticarcillin-clavulanic (TCC, 75/10 $\mu \mathrm{g}$ ) & 36 & Chloramphenicol (CHL, $30 \mu \mathrm{g})$ & 29 \\
\hline CEPHALOSPORIN CLASS & & LINCOSAMIDES CLASS & \\
\hline Cephalexin (CEF, $30 \mu \mathrm{g})$ & 30 & Lincomycin LCN, $15 \mu \mathrm{g})$ & 13 \\
\hline Cefuroxime (CXM, $30 \mu \mathrm{g})$ & 24 & Clindamycin (CMN, $2 \mu \mathrm{g})$ & 6 \\
\hline Cefixime (CFM, $10 \mu \mathrm{g})$ & 28 & QUINOLONES CLASS & \\
\hline Ceftazidine $(\mathrm{CAZ}, 30 \mu \mathrm{g})$ & 21 & Pefloxacin (PEF, $5 \mu \mathrm{g})$ & 0 \\
\hline Cefpodoxine (CPD, $10 \mu \mathrm{g})$ & 19 & Ciprofloxacin (CIP, $5 \mu \mathrm{g})$ & 0 \\
\hline Ceftriaxone $(\mathrm{CFO}, 30 \mu \mathrm{g})$ & 31 & Nalidixic acid (NAL, $30 \mu \mathrm{g}$ ) & 0 \\
\hline AMINOGLYCOSIDES CLASS & & ANSAMYCIN CLASS & \\
\hline Gentamicin (GMI, 15 $\mu \mathrm{g})$ & 0 & Rifampycin (RAM, $30 \mu \mathrm{g})$ & 27 \\
\hline CARBAPENEM CLASS & & GLYCOPEPTIDES CLASS & \\
\hline \multirow[t]{2}{*}{ Imipenem (IPM, $10 \mu \mathrm{g})$} & 25 & Vancomycin (VAN, $30 \mu \mathrm{g})$, & 19 \\
\hline & & Teicoplanin (TEC, $30 \mu \mathrm{g}$ ) & 15 \\
\hline
\end{tabular}




\section{A) $25^{\circ} \mathrm{C}$, without SVF}

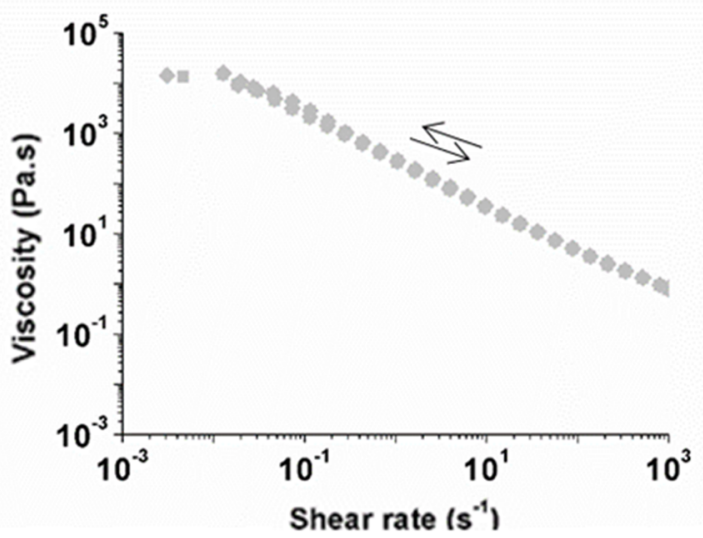

\section{C) without SVF}

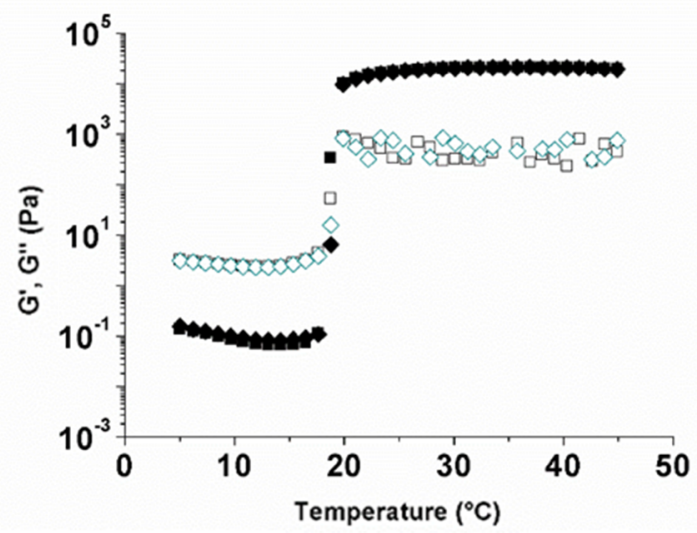

E) $25^{\circ} \mathrm{C}$, without SVF

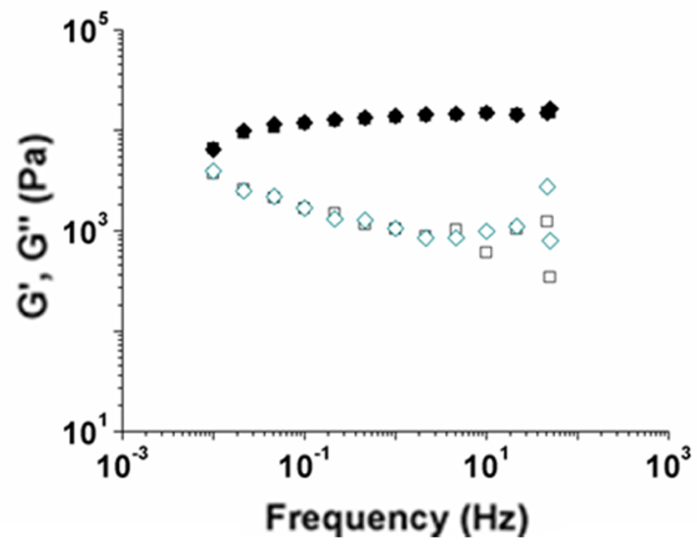

B) $37^{\circ} \mathrm{C}$, with SVF

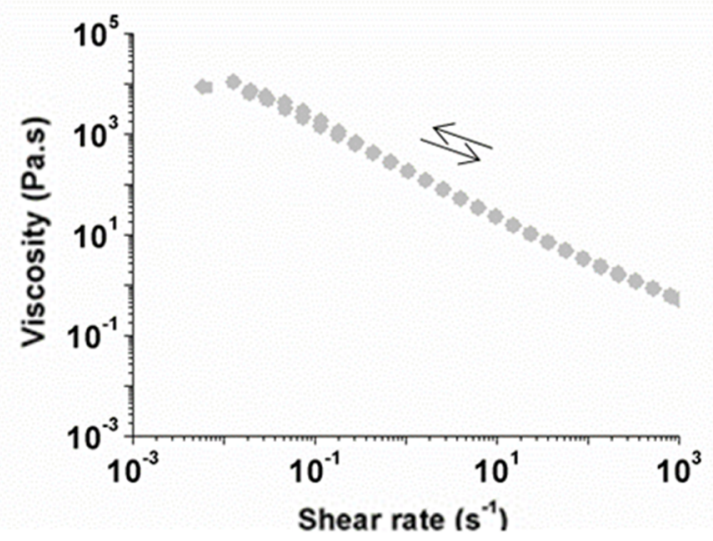

D) with SVF

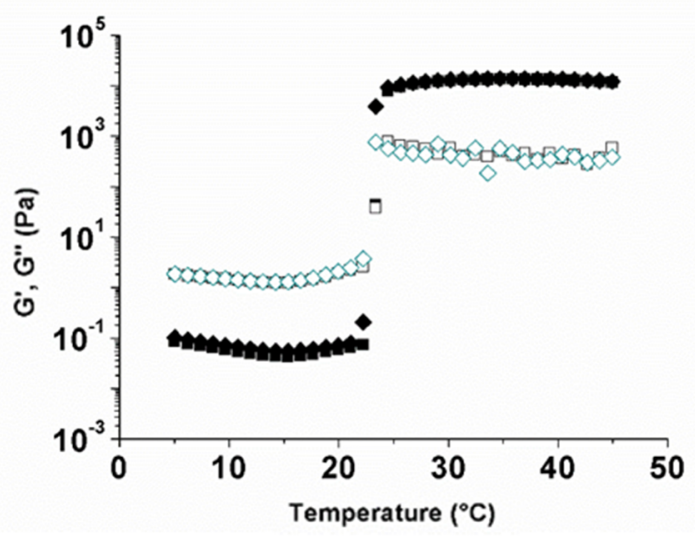

F) $37^{\circ} \mathrm{C}$ with SVF

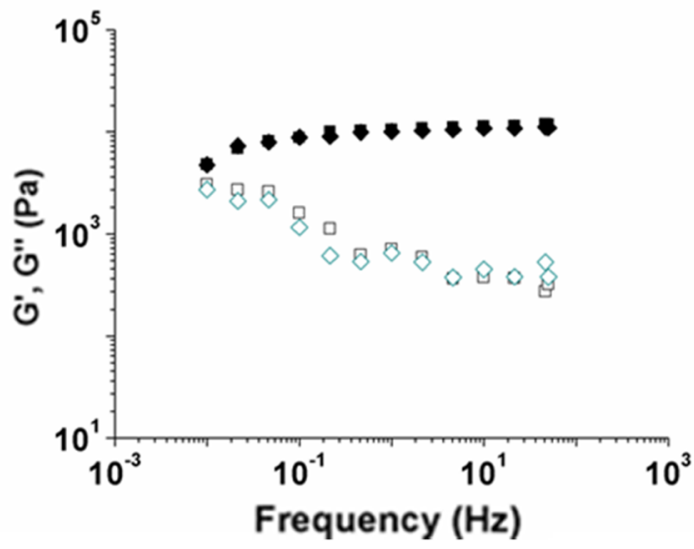

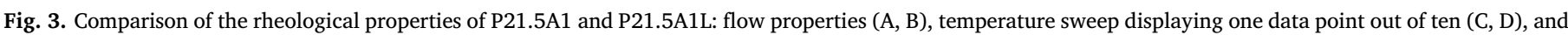

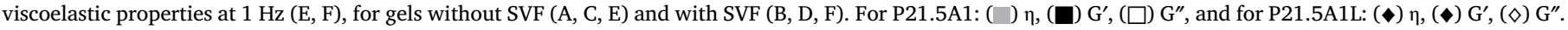
Note that P21.5A1 and P21.5A1L curves are superimposed.

\subsection{Distribution of L. crispatus within the gel}

For further characterization of the gel containing L. crispatus, the distribution of $L$. crispatus immediately after the gel preparation was studied by confocal fluorescence microscopy (CLSM), using both Syto 9 and propidium iodide (Fig. 4). Zotta et al. (2012) showed the usefulness of two stain indicators for optimal results in the evaluation of cells viability. In the present study, Syto9 was used to stain live bacteria in 
Table 4

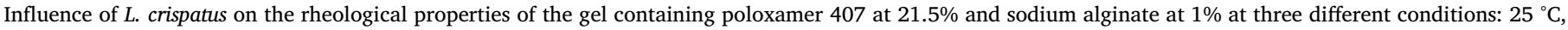
$37^{\circ} \mathrm{C}$ and $37^{\circ} \mathrm{C}$ after dilution with SVF.

\begin{tabular}{|c|c|c|c|c|c|}
\hline \multirow[t]{2}{*}{ Samples } & \multirow[t]{2}{*}{$\mathrm{T}_{\text {gel }}\left({ }^{\circ} \mathrm{C}\right)$} & \multicolumn{3}{|c|}{ Viscoelastic properties at $1 \mathrm{~Hz}$} & \multirow[t]{2}{*}{ Flow properties } \\
\hline & & Conditions & $\mathrm{G}^{\prime}\left(10^{3} \mathrm{~Pa}\right)$ & $\mathrm{G}^{\prime \prime}\left(10^{3} \mathrm{~Pa}\right)$ & \\
\hline \multirow{2}{*}{ P21.5A1 } & $19 \pm 1$ & $25^{\circ} \mathrm{C}$ & $13.5 \pm 0.4$ & $1.0 \pm 0.2$ & Shear-thinning \\
\hline & & $37^{\circ} \mathrm{C}$ & $17.6 \pm 0.5$ & $0.5 \pm 0.6$ & Non- thixotropic \\
\hline $\mathrm{P} 21.5 \mathrm{~A} 1+\mathrm{SVF}$ & $24 \pm 1$ & $37^{\circ} \mathrm{C}+\mathrm{SVF}$ & $10.5 \pm 0.2$ & $0.7 \pm 0.2$ & \\
\hline \multirow[t]{2}{*}{ P21.5A1L } & $19 \pm 1$ & $25{ }^{\circ} \mathrm{C}$ & $13.9 \pm 0.3$ & $1.1 \pm 0.5$ & \\
\hline & & $37^{\circ} \mathrm{C}$ & $16.5 \pm 0.4$ & $0.7 \pm 0.1$ & \\
\hline $\mathrm{P} 21.5 \mathrm{~A} 1 \mathrm{~L}+\mathrm{SVF}$ & $23 \pm 1$ & $37^{\circ} \mathrm{C}+$ SVF & $10.1 \pm 0.3$ & $0.7 \pm 0.2$ & \\
\hline
\end{tabular}

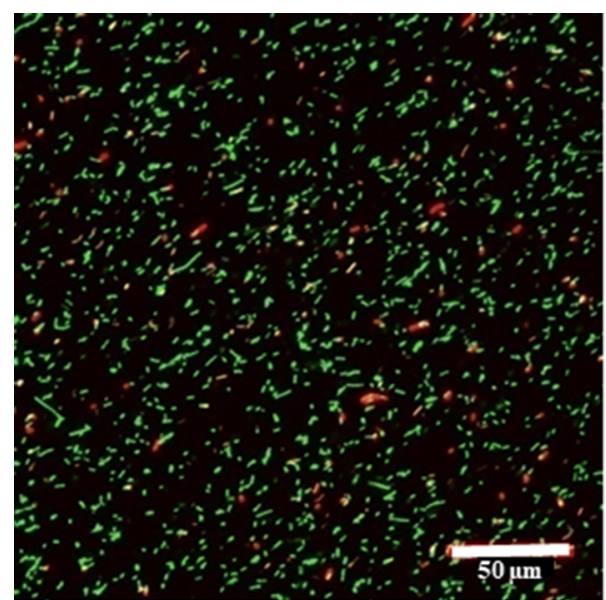

Fig. 4. Representative confocal microscopy image of the distribution of $L$. crispatus within the P21.5A1L hydrogel immediately after their preparation, showing a large majority of $L$. crispatus in green (living bacteria). Dead bacteria appear in red. Images correspond to the stacking of 37 images collected along the z-axis. (For interpretation of the references to color in this figure legend, the reader is referred to the web version of this article.)

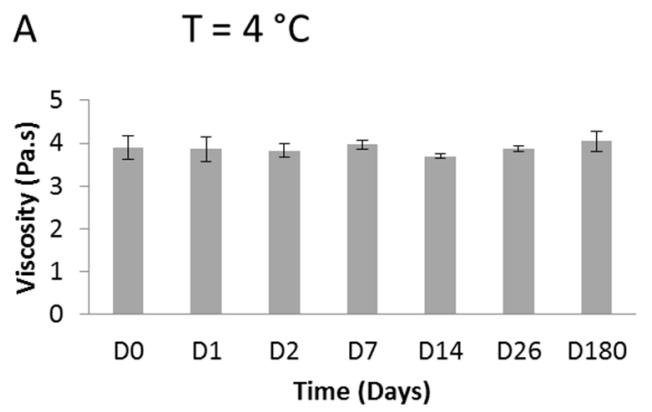

C $\quad \mathrm{T}=4{ }^{\circ} \mathrm{C}$

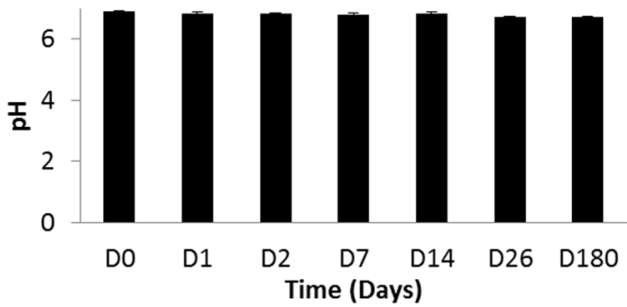

green whereas propidium iodide was used to stain dead cells. Almost all the $L$. crispatus cells were green stained confirming their viability after their introduction into the gel. The distribution seemed to be similar at all the observed points, from two different wells of the same gel. Thus, CLSM showed a homogeneous distribution of $L$. crispatus in the gel along the $\mathrm{x}, \mathrm{y}$ and $\mathrm{z}$ axes (Fig. 4). This distribution of L. crispatus was repeatable and reproducible, showing the reproducibility of the method of gel preparation. This homogeneous distribution of $L$. crispatus in the gel should be favorable to a homogeneous distribution of $L$. crispatus in the vagina after administration.

\subsection{Influence of the storage conditions}

The stability of the gel P21.5A1L was examined for 6 months at two different storage conditions: 4 and $25{ }^{\circ} \mathrm{C}$. Viscosity of the gel (Fig. 5A, B) as well as its $\mathrm{pH}$ (Fig. 5C, D) remained unchanged over these 6 months of storage at both temperatures (Fig. 5A, C and Fig. 5B, D). However, the viability and the culturability of formulated $L$. crispatus were modified (Figs. 6 and 7). The number of $L$. crispatus assessed by counting on MRS agar plate decreased drastically after 14 days of storage at $4{ }^{\circ} \mathrm{C}$ (Fig. 6) and after 2 days at $25^{\circ} \mathrm{C}$ (Fig. 7). The number of $L$. crispatus was assessed by confocal microscopy using Syto 9 and propidium iodide (Fig. 6). Samples were analysed by two types of acquisition, the z-stack acquisition of two different areas and the single stack of

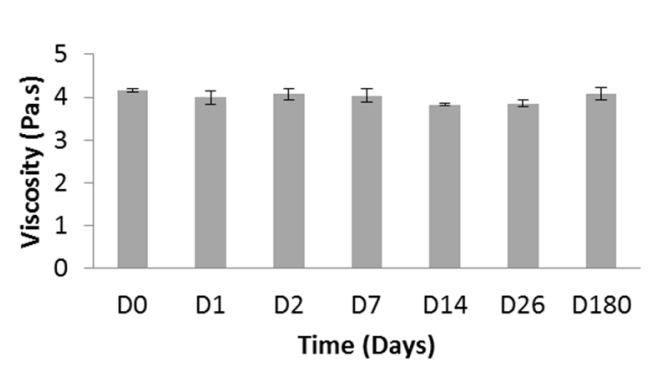

D
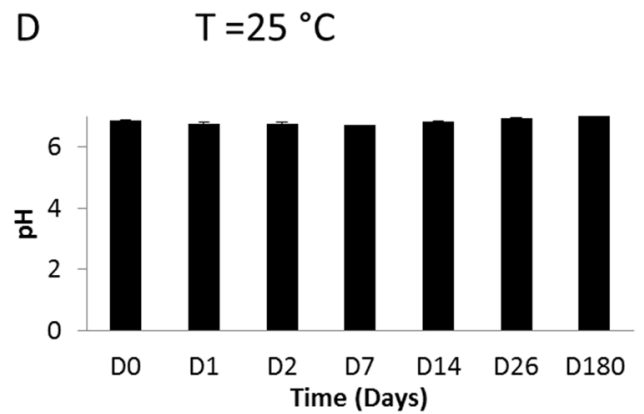

Fig. 5. Viscosity (A, B) and pH (C, D) measurements of a gel containing poloxamer 407 at $21.5 \%$, sodium alginate at $1 \%$ and $L$. crispatus at $9 \log _{10} \mathrm{CFU}$ per gel unit (5 g), during its storage over six months at $4{ }^{\circ} \mathrm{C}(\mathrm{A}, \mathrm{C})$ or $25^{\circ} \mathrm{C}(\mathrm{B}, \mathrm{D})$. 


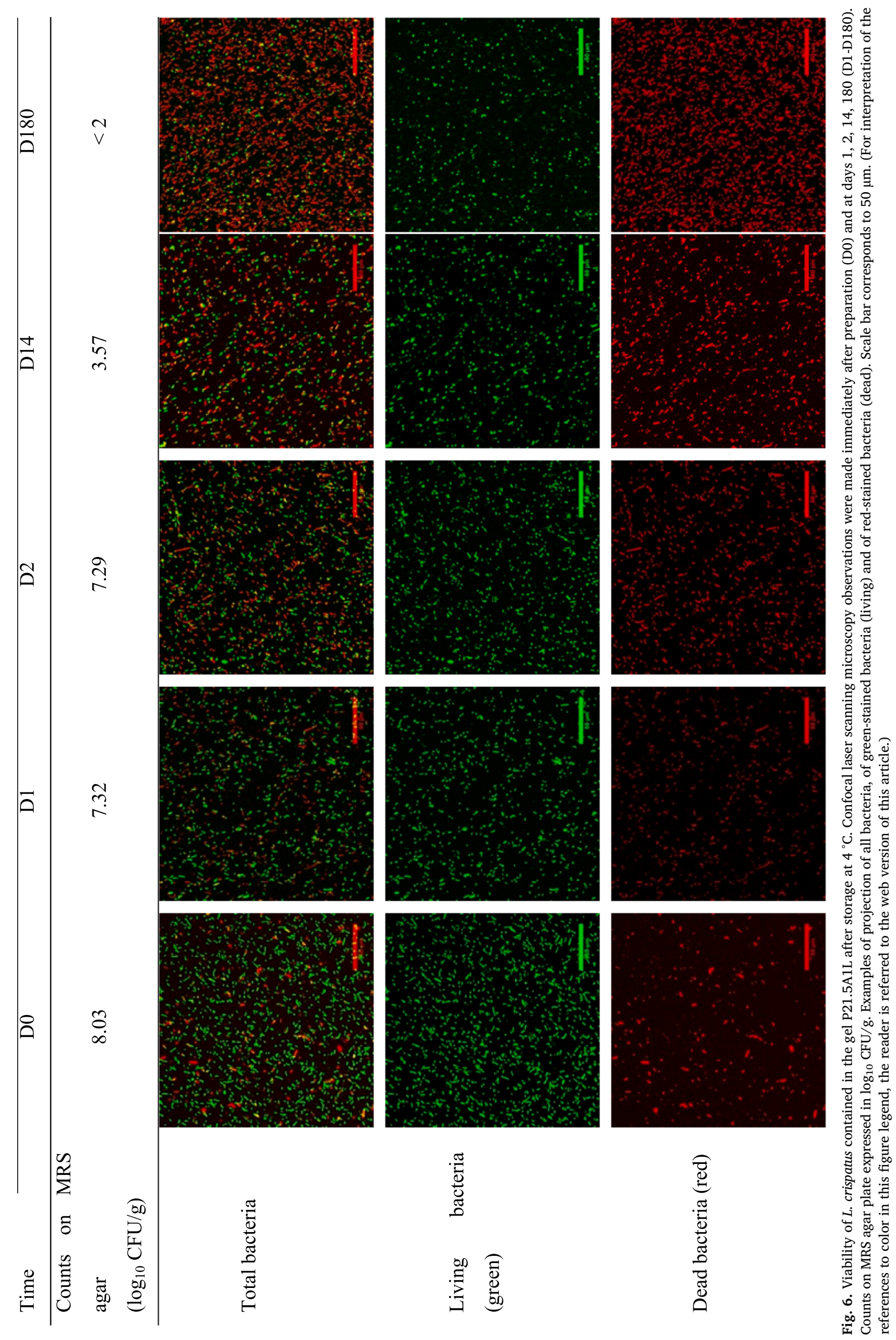




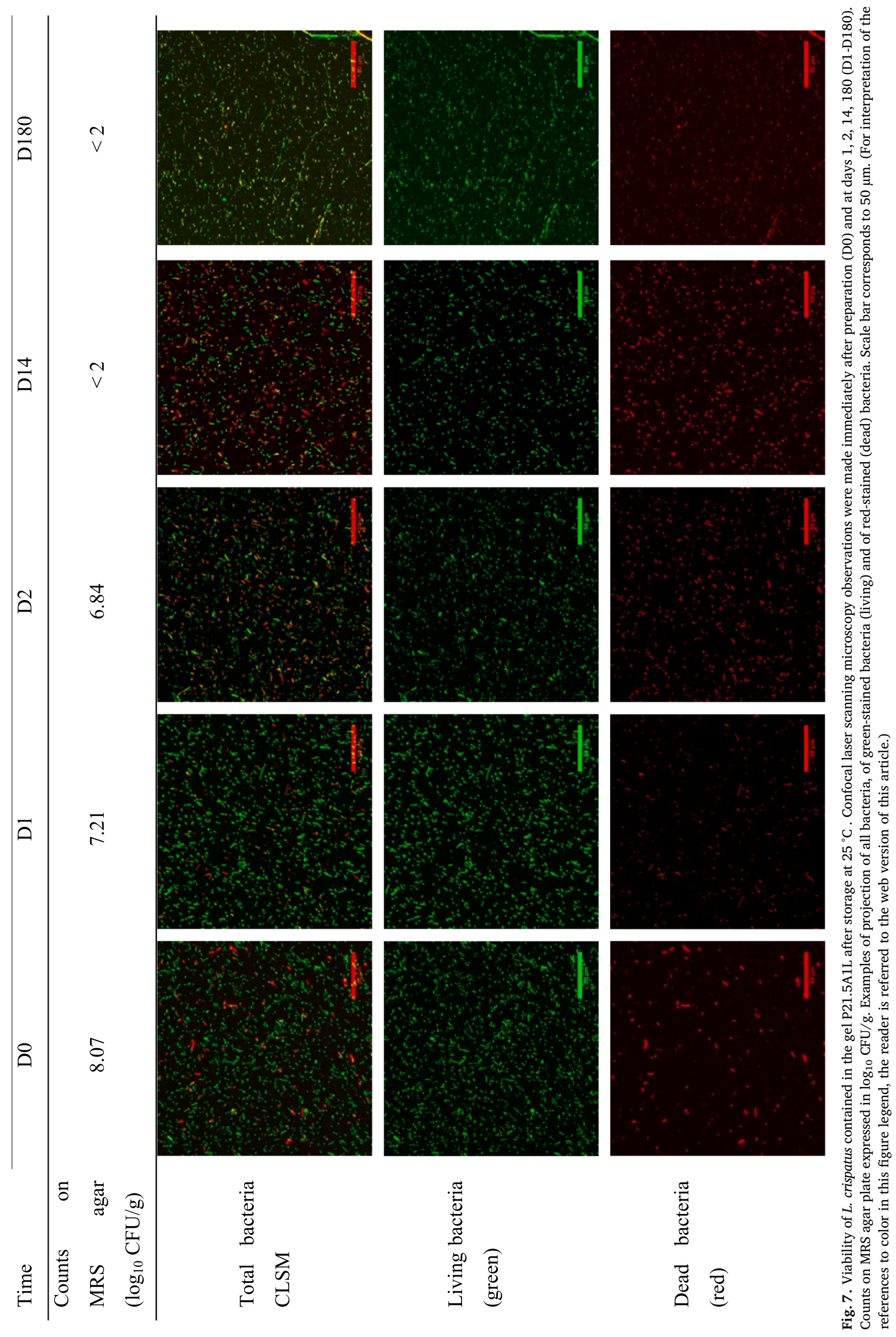


eight different areas. The coefficient of correlation between the two types of image acquisition was 0.93 . Despite the observation of greenlabeled bacteria on the images of the confocal microscopy, there was no bacterial count by agar count method on MRS plate at D180 (storage at $4{ }^{\circ} \mathrm{C}$ ) and at D14 and D180 (storage at $25{ }^{\circ} \mathrm{C}$ ). This result suggested the development of viable but nonculturable (VNC) bacteria in the gel during storage at both $4{ }^{\circ} \mathrm{C}$ and $25^{\circ} \mathrm{C}$. This can be due to the absence of nutrient inducing a stress of $L$. crispatus in the gel. Indeed, the enumeration by agar plate method often leads to an underestimation of bacterial total number due to the formation of cells chains and clumps or to the induction of VNC state after an exposure to stress or starvation (Léonard et al., 2015; Rault et al., 2007; Zotta et al., 2009). These results are in accordance with several studies which showed that cell viability estimated by microscopy was higher than viability assessed by plate counts. VNC cells can not be cultured using standard microbiological media because they are incapable of undergoing the sustained cellular division required to form a colony on regular agar media but they often maintain a potential for metabolic activity at the single cell level (Zotta et al., 2009).

As the number of viable $L$. crispatus decreased in gel during storage, further studies are needed to preserve its viability over time. In this respect, hydrogel lyophilization, addition of prebiotics in the formulation, or extemporaneous mixing of gel and L. crispatus might be interesting strategies.

\subsection{Efficacy of inhibition of N. gonorrhoeae}

The ability of $L$. crispatus to inhibit the growth of $N$. gonorrhoeae in vitro, was asssed at two different $\mathrm{pH}(5,6.5)$ (Table 5). In the absence of L. crispatus, $N$. gonorrhoeae grew onto chocolate agar at $\mathrm{pH} 6.5$ but did not grow onto this media at $\mathrm{pH}$ 5.0. The inhibition of $N$. gonorrhoeae was due to the acidic environment. After deposition of spots of $L$. crispatus suspension onto BHIA at $\mathrm{pH}$ 6.5, an inhibition zone of N. gonorrhoeae was clearly observed. These results were in accordance with those of Foschi et al. (2017), who demonstrated that the L. crispatus strains they studied were also able to counteract $N$. gonorrhoeae viability through multiple mechanisms (acidic environment creation, lactate production, biosurfactant production and co-aggregation), and could represent a new potential probiotic strategy for the prevention of gonorrhea in women.

The gel P21.5A1 alone had no effect on $N$. gonorrhoeae growth. Conversely, at pH 6.5, the P21.5A1L gel containing L. crispatus inhibited the growth of pathogens (Table 5) with an efficacy depending on $L$. crispatus load. L. crispatus was thus responsible of the inhibition by the gel at $\mathrm{pH}$ 6.5. When using BHIA $\mathrm{pH} 6.5$, the inhibition zone of the suspension containing $8 \log _{10}$ L. crispatus was similar to the inhibition zone of the gel containing $9 \log _{10} L$. crispatus. The gel, due to its viscosity, could slow down the diffusion of substances that are produced by L. crispatus and are involved in the inhibition mechanisms. Despite this lower in vitro efficacy compared to a simple $L$. crispatus suspension, gels remain interesting formulations. Indeed, liquid suspensions are known to lead to a rapid flow and clearance after vaginal administration whereas the gel will have a higher residence time due to its viscosity and mucoadhesive properties. Otherwise, the method used to assess the inhibition of $N$. gonorrhoeae only explored the influence of the $\mathrm{pH}$ and the diffusion of inhibitory molecules through the agar layer $(\sim 2 \mathrm{~mm})$. Further studies by direct contact with $N$. gonorrhoeae should be performed to determine if $L$. crispatus, when incorporated in gel formulations, preserves all its inhibition properties on $N$. gonorrhoeae under the conditions prevailing in the vaginal cavity in vivo.

\section{Table 5}

Inhibition of the growth of $N$. gonorrhoeae in vitro by agar overlayed method, after $24 \mathrm{~h}$ of incubation at $37{ }^{\circ} \mathrm{C}, 5 \% \mathrm{CO}_{2}$. Comparative study of $L$. crispatus suspension and of gels P21.5A1 and P21.5A1L. Four different experiments (tests).

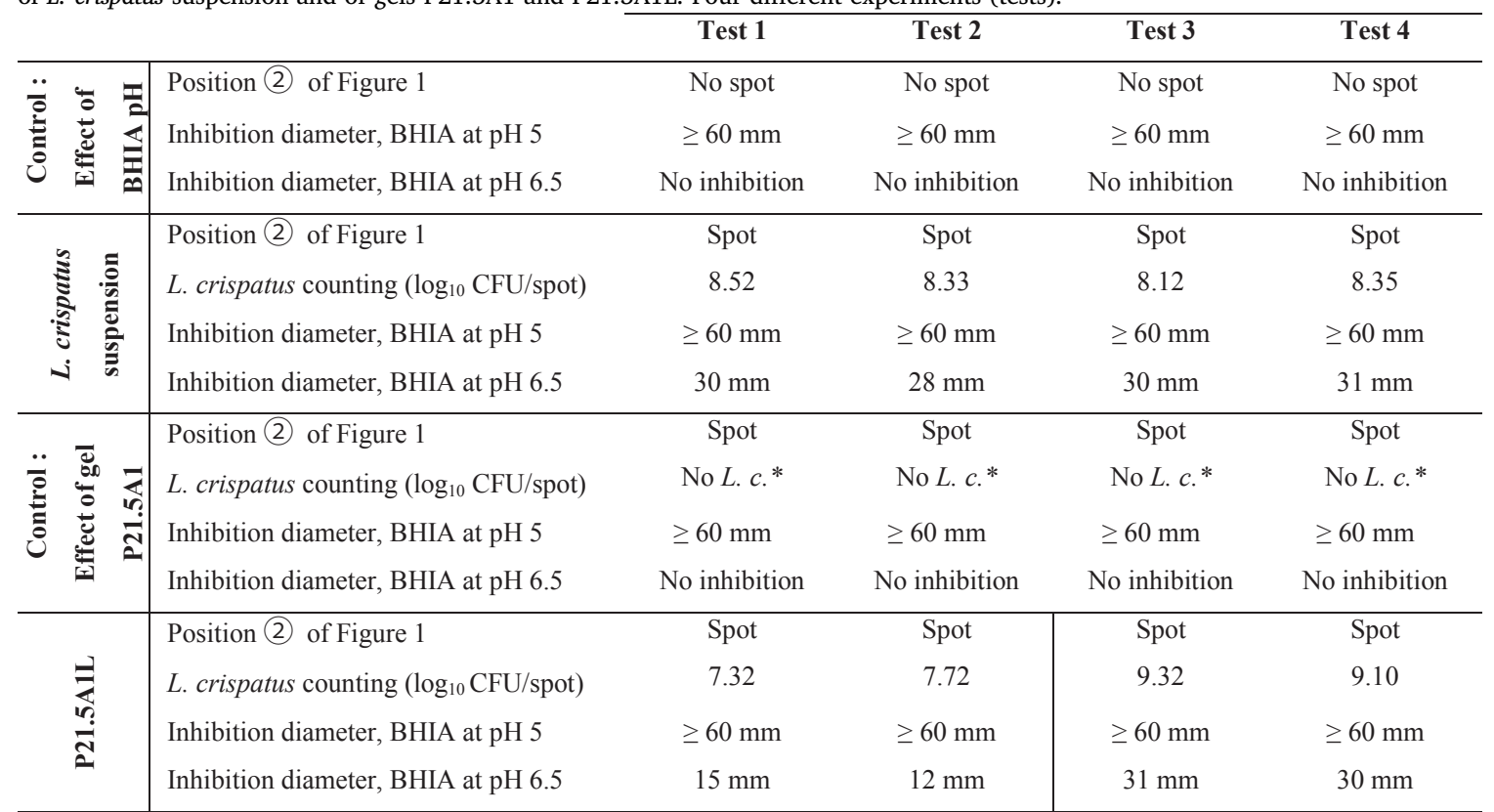

\footnotetext{
* L. C.: L. crispatus.
} 


\section{Conclusion}

This study showed the probiotic potential of L. crispatus ATCC 33197 and its ability to inhibit $N$. gonorrhoeae. L. crispatus can be considered as a good candidate to be used in the development of vaginal formulations for the prevention of gonorrhea. Gels containing poloxamer 407 at $21.5 \%$ and alginate at $1 \%$ with or without $L$. crispatus ATCC 33197 showed rheological properties favorable for a vaginal administration. Their process of preparation was not deleterious for the Lactobacilli cells. L. crispatus was able to inhibit $N$. gonorrhoeae even when incorporated in the gel. Therefore, this vaginal gel could be promising as a single-dose system intended to be administered to women at risk due to a history of vaginal infections and to women having naturally a deficiciency of lactobacilli in their vaginal flora. Such treatment might limit gonorrhoea transmission in women that do not have the possibility to negotiate the use of condom during sexual intercourses. Yet, L. crispatus viability should be optimized by lyophilization or the addition of some nutrients in the formulation and in vivo studies should also be conducted to evaluate the efficacy and the safety of the gel. The number, frequency and timing of administration, as well as the effect of sexual intercourses, are important parameters to assess in further clinical studies.

\section{CRediT authorship contribution statement}

K.C. N'Guessan Gnaman: Conceptualization, Methodology, Investigation, Visualization, Writing - original draft. S. Bouttier: Conceptualization, Methodology, Investigation, Writing - review \& editing. A. Yeo: Conceptualization, Methodology, Writing - review \& editing. A.A.S. Aka Any-Grah: Conceptualization, Supervision, Writing - review \& editing. S. Geiger: Conceptualization, Supervision, Writing review \& editing. N. Huang: Methodology, Writing - review \& editing. V. Nicolas: Methodology, Writing - review \& editing. S. Villebrun: Investigation. H. Faye-Kette: Conceptualization, Writing - review \& editing. G. Ponchel: Conceptualization, Writing - review \& editing. A.A. Koffi: Conceptualization, Supervision, Writing - review \& editing, Funding acquisition, Project administration. F. Agnely: Conceptualization, Supervision, Writing - review \& editing, Funding acquisition, Project administration.

\section{Declaration of Competing Interest}

The authors declare that they have no known competing financial interests or personal relationships that could have appeared to influence the work reported in this paper.

\section{Acknowledgements}

This work was supported by Institut de recherche et de développement (IRD), Franco-Ivorian cooperation, Campus France, AMRUGE-CI/C2D grant (support to the modernization and reform of universities and colleges of Côte d'Ivoire). The authors thank the region île-de-France for the purchase of the confocal microscope and are grateful to Luc Picton and Christophe Rihouey from Laboratoire Polymères, Biopolymères, Surfaces, UMR 6270 CNRS, Université de Rouen, for the determination of alginate molecular weight and Charles Konan Kouassi from Université Abobo-Adjamé for his technical help during optical microscopy observations. Clémence N'Guessan acknowledges Université Paris-Saclay for her international dual degree PhD grant.

\section{References}

Aka-Any-Grah, A., Bouchemal, K., Koffi, A., Agnely, F., Zhang, M., Djabourov, M., Ponchel, G., 2010. Formulation of mucoadhesive vaginal hydrogels insensitive to dilution with vaginal fluids. Eur. J. Pharm. Biopharm. 76, 296-303.
Amant, D.C.S., Valentin-Bon, I.E., Jerse, A.E., 2002. Inhibition of Neisseria gonorrhoeae by Lactobacillus species that are commonly isolated from the female genital tract. Infect. Immun. 70, 7169-7171.

Ambalam, P., Ramoliya, J.M., Dave, J.M., Vyas, B.R.M., 2012. Safety assessment of potential probiotic strains Lactobacillus rhamnosus 231 and Lactobacillus rhamnosus v92 in mouse model. Int. J. Bioassays 2, 333-337.

Anderson, M.T., Byerly, L., Apicella, M.A., Seifert, H.S., 2016. Seminal plasma promotes Neisseria gonorrhoeae aggregation and biofilm formation. J. Bacteriol. 198, 2228-2235.

Antonio, M.A.D., Hillier, S.L., 2003. DNA fingerprinting of Lactobacillus crispatus strain CTV-05 by repetitive element sequence-based PCR analysis in a pilot study of vaginal colonization. J. Clin. Microbiol. 41, 1881-1887.

Antonio, M.A.D., Hawes, S.E., Hillier, S.L., 1999. The identification of vaginal Lactobacillus species and the demographic and microbiologic characteristics of women colonized by these species. J. Infect. Dis. 180, 1950-1956.

Barnhart, K.T., Pretorius, E.S., Timbers, K., Shera, D., Shabbout, M., Malamud, D., 2004 In vivo distribution of a vaginal gel: MRI evaluation of the effects of gel volume, time and simulated intercourse. Contraception 70, 498-505.

Boskey, E.R., Telsch, K.M., Whaley, K.J., Moench, T.R., Cone, R.A., 1999. Acid production by vaginal flora in vitro is consistent with the rate and extent of vaginal acidification. Infect. Immun. 67, 5170-5175.

Breshears, L.M., Edwards, V.L., Ravel, J., Peterson, M.L., 2015. Lactobacillus crispatus inhibits growth of Gardnerella vaginalis and Neisseria gonorrhoeae on a porcine vaginal mucosa model. BMC Microbiol. 15.

Bruce, A.W., Reid, G., 1988. Intravaginal instillation of Lactobacilli for prevention of recurrent urinary tract infections. Can. J. Microbiol. 34, 339-343.

Caramella, C.M., Rossi, S., Ferrari, F., Bonferoni, M.C., Sandri, G., 2015. Mucoadhesive and thermogelling systems for vaginal drug delivery. Adv. Drug Deliv. Rev. 92, 38-52.

Chaluvadi, S., Hotchkiss Jr., A.T., Call, J.E., Luchansky, J.B., Phillips, J.G., Liu, L., Yam, K.L., 2012. Protection of probiotic bacteria in a synbiotic matrix following aerobic storage at $4{ }^{\circ} \mathrm{C}$. Benef. Microbes 3, 175-187.

Cohen, M.S., 2004. HIV and sexually transmitted diseases: lethal synergy. Top. HIV Med. Publ. Int. AIDS Soc. U.S.A. 12, 104-107.

Cook, M.T., Brown, M.B., 2018. Polymeric gels for intravaginal drug delivery. J. Control. Release 270, 145-157.

Czaja, C.A., Stapleton, A.E., Yarova-Yarovaya, Y., Stamm, W.E., 2007. Phase I trial of a Lactobacillus crispatus vaginal suppository for prevention of recurrent urinary tract infection in women. Infect. Dis. Obstet. Gynecol. 2007, 35387. https://doi.org/10. $1155 / 2007 / 35387$

Danielsen, M., Wind, A., 2003. Susceptibility of Lactobacillus spp. to antimicrobial agents. Int. J. Food Microbiol. 82, 1-11.

Dumortier, G., Grossiord, J.L., Agnely, F., Chaumeil, J.C., 2006. A review of poloxamer 407 pharmaceutical and pharmacological characteristics. Pharm. Res. 23, 2709-2728.

Foschi, C., Salvo, M., Cevenini, R., Parolin, C., Vitali, B., Marangoni, A., 2017. Vaginal Lactobacilli reduce Neisseria gonorrhoeae viability through multiple strategies: an in vitro study. Front. Cell. Infect. Microbiol. 7.

Giuliano, E., Paolino, D., Fresta, M., Cosco, D., 2018. Mucosal applications of Poloxamer 407-based hydrogels: an overview. Pharmaceutics 10, 159.

Grassi, G., Crevatin, A., Farra, R., Guarnieri, G., Pascotto, A., Rehimers, B., Lapasin, R., Grassi, M., 2006. Rheological properties of aqueous Pluronic-alginate systems containing liposomes. J. Colloid Interface Sci. 301, 282-290.

Graver, M.A., Wade, J.J., 2011. The role of acidification in the inhibition of Neisseria gonorrhoeae by vaginal Lactobacilli during anaerobic growth. Ann. Clin. Microbiol. Antimicrob. 10, 8.

Grodstein, F., Goldman, M.B., Cramer, D.W., 1993. Relation of tubal infertility to history of sexually transmitted diseases. Am. J. Epidemiol. 137, 577-584.

Hemmerling, A., Harrison, W., Schroeder, A., Park, J., Korn, A., Shiboski, S., FosterRosales, A., Cohen, C.R., 2010. Phase 2a study assessing colonization efficiency, safety, and acceptability of Lactobacillus crispatus CTV-05 in women with bacterial vaginosis. Sex. Transm. Dis. 37, 745-750.

Hill, C., Guarner, F., Reid, G., Gibson, G.R., Merenstein, D.J., Pot, B., Morelli, L., Canani, R.B., Flint, H.J., Salminen, S., et al., 2014. Expert consensus document. The International Scientific Association for Probiotics and Prebiotics consensus statement on the scope and appropriate use of the term probiotic. Nat. Rev. Gastroenterol. Hepatol. 11, 506-514.

Huang, L., Shen, M., Li, R., Zhang, X., Sun, Y., Gao, P., Fu, H., Liu, H., He, Y., Du, Y., et al., 2016. Thermo-sensitive composite hydrogels based on poloxamer 407 and alginate and their therapeutic effect in embolization in rabbit VX2 liver tumors. Oncotarget 7, 73280 .

Jarvis, G.A., Chang, T.L., 2012. Modulation of HIV transmission by Neisseria gonorrhoeae: molecular and immunological aspects. Curr. HIV Res. 10, 211-217.

Kassaa, I.A., Hamze, M., Hober, D., Chihib, N.-E., Drider, D., 2014. Identification of vaginal Lactobacilli with potential probiotic properties isolated from women in north Lebanon. Microb. Ecol. 67, 722-734.

Laga, M., Plummer, F.A., Nzanze, H., Namaara, W., Brunham, R.C., Ndinya-Achola, J.O., Maitha, G., Ronald, A.R., D'Costa, L.J., Bhullar, V.B., 1986. Epidemiology of ophthalmia neonatorum in Kenya. The Lancet 2, 1145-1149.

Lee, K.Y., Mooney, D., 2012. Alginate: properties and biomedical applications. Prog. Polym. Sci. 37, 106-126.

Lefebvre, B., Martin, I., Demczuk, W., Deshaies, L., Michaud, S., Labbé, A.-C., Beaudoin, M.-C., Longtin, J., 2018. Ceftriaxone-resistant Neisseria gonorrhoeae, Canada, 2017. Emerg. Infect. Dis. 24 (2), 381-383.

Léonard, L., Beji, O., Arnould, C., Noirot, E., Bonnotte, A., Gharsallaoui, A., Degraeve, P., Lherminier, J., Saurel, R., Oulahal, N., 2015. Preservation of viability and anti- 
Listeria activity of lactic acid bacteria, Lactococcus lactis and Lactobacillus paracasei, entrapped in gelling matrices of alginate or alginate/caseinate. Food Control 47, 7-19.

Lin, H.-R., Sung, K.C., Vong, W.-J., 2004. In situ gelling of alginate/pluronic solutions for ophthalmic delivery of pilocarpine. Biomacromolecules 5, 2358-2365.

Liu, Y., Zhu, Y., Wei, G., Lu, W., 2009. Effect of carrageenan on poloxamer-based in situ gel for vaginal use: improved in vitro and in vivo sustained-release properties. Eur. J. Pharm. Sci. 37, 306-312.

Maragkoudakis, P.A., Zoumpopoulou, G., Miaris, C., Kalantzopoulos, G., Pot, B., Tsakalidou, E., 2006. Probiotic potential of Lactobacillus strains isolated from dairy products. Int. Dairy J. 16, 189-199.

Maragkoudakis, P.A., Papadelli, M., Georgalaki, M., Panayotopoulou, E.G., MartinezGonzalez, B., Mentis, A.F., Petraki, K., Sgouras, D.N., Tsakalidou, E., 2009. In vitro and in vivo safety evaluation of the bacteriocin producer Streptococcus macedonicus ACA-DC 198. Int. J. Food Microbiol. 133, 141-147.

Minnis, A.M., Padian, N.S., 2005. Effectiveness of female controlled barrier methods in preventing sexually transmitted infections and HIV: current evidence and future research directions. Sex. Transm. Infect. 81, 193-200.

Moore, S., Kailasapathy, K., Phillips, M., Jones, M.R., 2015. Development of a viability standard curve for microencapsulated probiotic bacteria using confocal microscopy and image analysis software. J. Microbiol. Methods 114, 16-22.

Nader-Macias, M.E., Juarez Tomas, M.S., 2015. Profiles and technological requirements of urogenital probiotics. Adv. Drug Deliv. Rev. 92, 84-104.

O'Hanlon, D.E., Moench, T.R., Cone, R.A., 2013. Vaginal pH and microbicidal lactic acid when Lactobacilli dominate the microbiota. PLoS ONE 8, e80074.

Owen, D.H., Katz, D.F., 1999. A vaginal fluid simulant. Contraception 59, 91-95.

Palmeira-de-Oliveira, R., Palmeira-de-Oliveira, A., Martinez-de-Oliveira, J., 2015. New strategies for local treatment of vaginal infections. Adv. Drug Deliv. Rev. 92, 105-122.

Pavlova, S.I., Kilic, A.O., Kilic, S.S., So, J.-S., Nader-Macias, M.E., Simoes, J.A., Tao, L. 2002. Genetic diversity of vaginal lactobacilli from women in different countries based on 16S rRNA gene sequences. J. Appl. Microbiol. 92, 451-459.

Pilcher, H., 2004. Starting to gel. Nature 430, 138-140.

Querobino, S.M., de Faria, N.C., Vigato, A.A., da Silva, B.G.M., Machado, I.P., Costa, M.S., Costa, F.N., de Araujo, D.R., Alberto-Silva, C., 2019. Sodium alginate in oil-poloxamer organogels for intravaginal drug delivery: Influence on structural parameters, drug release machanisms, cytotoxicity and in vitro antifungal activity. Mater. Sci. Eng., C 99, 1350-1361.

Rault, A., Béal, C., Ghorbal, S., Ogier, J.-C., Bouix, M., 2007. Multiparametric flow cytometry allows rapid assessment and comparison of lactic acid bacteria viability after freezing and during frozen storage. Cryobiology 55, 35-43.

Ravel, J., Gajer, P., Abdo, Z., Schneider, G.M., Koenig, S.S., Stacey, L.M., Karlebach, S., Gorle, R., Russell, J., Carol, O.T., et al., 2011. Vaginal microbiome of reproductiveage women. PNAS 108, 4680-4687.

Reid, G., McGroarty, J.A., Domingue, P.A.G., Chow, A.W., Bruce, A.W., Eisen, A., Costerton, J.W., 1990. Coaggregation of urogenital bacteria in vitro and in vivo. Curr. Microbiol. 20, 47-52.

Reid, G., Beuerman, D., Heinemann, C., Bruce, A.W., 2001. Probiotic Lactobacillus dose required to restore and maintain a normal vaginal flora. FEMS Immunol. Med. Microbiol. 32, 37-41.
Rençber, S., Karavana, S.Y., Şenyiğit, Z.A., Eraç, B., Limoncu, M.H., Baloğlu, E., 2017 Mucoadhesive in situ gel formulation for vaginal delivery of clotrimazole: formulation, preparation, and in vitro/in vivo evaluation. Pharm. Dev. Technol. 22, 551-561.

Soliman, G.M., Fetih, G., Abbas, A.M., 2017. Thermosensitive bioadhesive gels for the vaginal delivery of sildenafil citrate: in vitro characterization and clinical evaluation in women using clomiphene citrate for induction of ovulation. Drug Dev. Ind. Pharm. 43 (3), 399-408.

Stapleton, A.E., Au-Yeung, M., Hooton, T.M., Fredricks, D.N., Roberts, P.L., Czaja, C.A., Yarova-Yarovaya, Y., Fiedler, T., Cox, M., Stamm, W.E., 2011. Randomized, placebocontrolled phase 2 trial of a lactobacillus crispatus probiotic given intravaginally for prevention of recurrent urinary tract infection. Clin. Infect. Dis. 52, 1212-1217.

Stein, Z.A., 1990. HIV prevention: the need for methods women can use. Am. J. Public Health 80, 460-462.

Štšepetova, J., Taelma, H., Smidt, I., Hütt, P., Lapp, E., Aotäht, E., Mändar, R., 2017. Assessment of phenotypic and genotypic antibiotic susceptibility of vaginal Lactobacillus sp.. J. Appl. Microbiol. 123, 524-534.

Szekalska, M., Puciłowska, A., Szymańska, E., Ciosek, P., Winnicka, K., 2016. Alginate: current use and future perspectives in pharmaceutical and biomedical applications. Int. J. Polym. Sci. 2016. https://doi.org/10.1155/2016/7697031.

Tachedjian, G., Aldunate, M., Bradshaw, C.S., Cone, R.A., 2017. The role of lactic acid production by probiotic Lactobacillus species in vaginal health. Res. Microbiol. 168, $782-792$.

Temmerman, R., Pot, B., Huys, G., Swings, J., 2003. Identification and antibiotic susceptibility of bacterial isolates from probiotic products. Int. J. Food Microbiol. 81, $1-10$.

Tønnesen, H.H., Karlsen, J., 2002. Alginate in drug delivery systems. Drug Dev. Ind. Pharm. 28, 621-630.

Traore, Y.L., Chen, Y., Ho, E.A., 2018. Current state of microbicide development. Clin. Pharmacol. Ther. 104, 1074-1081.

Vasquez, A., Jakobsson, T., Ahrne, S., Forsum, U., Molin, G., 2002. Vaginal Lactobacillus flora of healthy Swedish women. J. Clin. Microbiol. 40, 2746-2749.

Verdenelli, M.C., Coman, M.M., Cecchini, C., Silvi, S., Orpianesi, C., Cresci, A., 2014. Evaluation of antipathogenic activity and adherence properties of human Lactobacillus strains for vaginal formulations. J. Appl. Microbiol. 116, 1297-1307.

Verstraelen, H., Verhelst, R., Claeys, G., De Backer, E., Temmerman, M., Vaneechoutte, M., 2009. Longitudinal analysis of the vaginal microflora in pregnancy suggests that L. crispatus promotes the stability of the normal vaginal microflora and that L. gasser and/or L. iners are more conducive to the occurrence of abnormal vaginal microflora. BMC Microbiol. 9, 116.

Vigani, B., Faccendini, A., Rossi, S., Sandri, G., Bonferoni, M.C., Grisoli, P., Ferrari, F. 2019. Development of a mucoadhesive in situ gelling formulation for the delivery of Lactobacillus gasseri into vaginal cavity. Pharmaceutics 11, 511.

WHO, 2016. WHO guidelines for the treatment of Neisseria gonorrhoeae.

Zotta, T., Parente, E., Ricciardi, A., 2009. Viability staining and detection of metabolic activity of sourdough lactic acid bacteria under stress conditions. World J. Microbiol. Biotechnol.

Zotta, T., Guidone, A., Tremonte, P., Parente, E., Ricciardi, A., 2012. A comparison of fluorescent stains for the assessment of viability and metabolic activity of lactic acid bacteria. World J. Microbiol. Biotechnol. 28, 919-927. 